\title{
Freeform Electronic and Photonic Landscapes in Hexagonal Boron Nitride
}

\author{
Nolan Lassaline, ${ }^{\dagger}$ Deepankur Thureja, ${ }^{\dagger+}$ Thibault Chervy, ${ }^{*}$ Daniel Petter, ${ }^{\dagger}$ \\ Puneet A. Murthy, ${ }^{*}$ Armin W. Knoll, ${ }^{*}$ and David J. Norris ${ }^{*},+$ \\ †Optical Materials Engineering Laboratory, Department of Mechanical and Process Engineering, \\ ETH Zurich, 8092 Zurich, Switzerland \\ Quantum Photonics Group, Department of Physics, ETH Zurich, 8092 Zurich, Switzerland

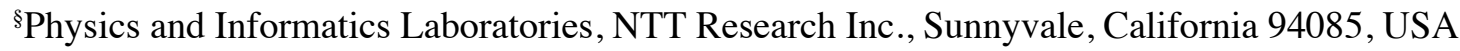 \\ ${ }^{\#}$ IBM Research - Zurich, 8803 Rueschlikon, Switzerland \\ *Email: dnorris@ethz.ch
}

\section{Contents}

Section

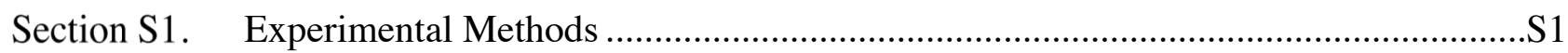

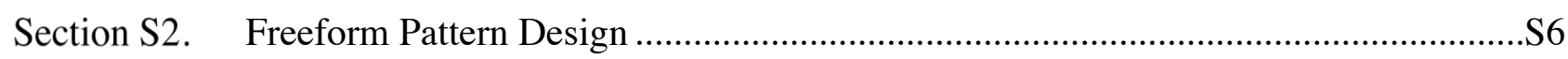

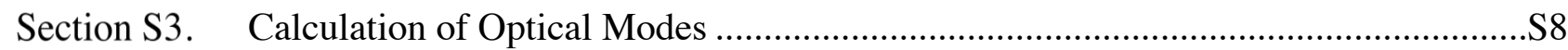

Section S4. Simulation of Electrostatic Potentials ................................................................

Section S5. Table of Experimental Design Parameters for Freeform Nanostructures ...............S10

Section S6. Supplementary Figures ….............................................................................

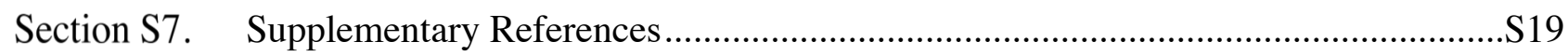

\section{Section S1. Experimental Methods}

Materials. Large-size bulk hBN crystals used in this work were purchased from 2D Semiconductors Inc., except the flake in Figure 3a, which was provided by T. Taniguchi and K. Watanabe. Silicon wafers with $285 \mathrm{~nm}$ of $\mathrm{SiO}_{2}$ were purchased from Alineason Materials Technology GmbH. For mechanical exfoliation of $\mathrm{hBN}$ we used adhesive tape purchased from Ultron Systems Inc. and 3M. The polymer resist for thermal scanning-probe lithography, PMMA/MA [AR-P 617, poly(methyl methacrylate-co-methacrylic acid), 33\% copolymer, diluted to either 1.5 or $3 \mathrm{wt} \%$ in 1-methoxy-2-propanol] was purchased from Allresist GmbH. 
Silicon cantilevers (MBS 2019-12) for thermal-scanning probe lithography were bought from Heidelberg Instruments Nano. Acetone and isopropyl alcohol (IPA) were provided by the Binnig and Rohrer Nanotechnology Center (BRNC) at IBM Zurich, where the fabrication was performed.

Sample fabrication. Silicon wafers with $285 \mathrm{~nm}$ of $\mathrm{SiO}_{2}$ were diced into chips of approximately $2 \times 2 \mathrm{~cm}^{2}$ area. Next, hBN flakes were deposited on the substrate using a mechanical exfoliation technique. ${ }^{\mathrm{S} 1, \mathrm{~S} 2}$ Briefly, repeated sticking and peeling of the hBN crystal to adhesive tape (Ultron Systems) thinned the bulk crystal down to flakes with thicknesses in the range of tens to hundreds of nanometers. After approximately 5-7 iterations of crystal thinning, Scotch-brand adhesive tape (3M) was applied to the crystal and removed. This tape with the thinned-down crystal was then placed on the $\mathrm{Si} / \mathrm{SiO}_{2}$ substrate (sticky side down) and a small vial filled with water was used as a weight to apply pressure to the top side of the tape. The substrate with tape (without weight) was moved onto a metal support, which was transferred to a hotplate, where it was heated for $1 \mathrm{~min}$ at $150^{\circ} \mathrm{C}$. After $1 \mathrm{~min}$, the metal support containing the sample was removed and allowed to cool for $1 \mathrm{~min}$. The tape was peeled off at a slow and uniform rate, leaving hBN flakes on the substrate ready for patterning.

PMMA/MA polymer resist was spin-coated onto the chip containing hBN flakes using a two-step procedure. The resist was dispensed onto the sample surface, which was then accelerated at 500 r.p.m. $\mathrm{s}^{-1}$ to 500 r.p.m. for 5 s. Next, the spinning speed was increased at 2000 r.p.m. ${ }^{-1}$ to the final spin speed (2000-6000 r.p.m., depending on the required thickness) for a total time of $40 \mathrm{~s}$. After spin-coating, the sample was baked at $180^{\circ} \mathrm{C}$ for $5 \mathrm{~min}$.

The sample was then placed on the stage of the thermal scanning-probe lithography tool (NanoFrazor Explore, Heidelberg Instruments Nano); the flake of interest was centered and rotationally aligned under the optical microscope of the tool. A cantilever was loaded into the cantilever holder, which was then attached to the Nanofrazor scan head. The tip was brought near the sample surface, and an automated approach function was used to find the sample surface and bring the tip into contact. For all freeform patterns other than the highresolution structures shown in Figure 2e and Figure 4, the tip was then moved away from the flake to perform calibration scans. After calibration, the tip was optically aligned over the flake of interest. Next, the thermal scanning probe performed a topography scan of the polymer surface on top of the flake for fine alignment of the pattern and to ensure that the surface was relatively flat and smooth in the local pattern area. The thermal scanning probe was set to an initial temperature of $950{ }^{\circ} \mathrm{C}$, and it then started fabricating the desired pattern, 
allowing the feedback to adjust the patterning conditions until the fabricated pattern matched the design pattern. The scan proceeded until the entire pattern was written in the polymer resist. Afterwards, the thermal scanning probe was available to create the next pattern on either the same flake or a different flake on the same chip.

For the high-resolution patterns, a fresh cantilever was loaded as in the procedure above, however, no calibration scans were performed. This minimized the contamination that builds up on the tip during patterning, which limits the resolution. The fresh cantilever was positioned directly over the flake of interest, and the patterning was initiated, but in this case the feedback was turned off. This was done to have maximum control over the patterning conditions. The starting temperature was set between $950-1000{ }^{\circ} \mathrm{C}$, and a minimal value of writing force was applied between the tip and substrate to observe conditions where no pattern was generated. The onset of patterning was initiated by repeatedly writing the same structure, where the write force was slowly increased for each scan until the desired pattern was observed in the polymer resist. Further adjustments to the temperature and write force were implemented manually and iteratively, until optimal conditions were found for the high-resolution pattern of interest. Once these conditions were identified, the patterns of interest were consecutively written in the polymer film. This typically resulted in a few tens of patterns before the cantilever had to be changed due to contamination build-up on the tip.

Once thermal scanning-probe lithography was completed, the pattern was transferred to the underlying hBN flake via inductively coupled plasma (ICP) etching ${ }^{\mathrm{S} 3}$ (Oxford Instruments, PlasmaPro) using a gas content of $50 \mathrm{sccm} \mathrm{SF}_{6}$. The etching was performed with a chamber pressure of $40 \mathrm{mTorr}$, a forward power of $75 \mathrm{~W}$, and at a rate of $\sim 2 \mathrm{~nm} \mathrm{~s}^{-1}$ until the polymer resist was removed. The pattern was transferred to the underlying hBN with a slight amplification $(\sim 1.5 \times)$ in the pattern depth compared to that in the polymer. After etching, the sample was sonicated for $2 \mathrm{~min}$ in acetone, rinsed with IPA, and blown dry with $\mathrm{N}_{2}$ gas.

Surface-topography characterization. The topography of the patterns in the polymer resist was measured by the thermal scanning probe during the writing process. The final patterns in $\mathrm{hBN}$ were measured with an AFM (Bruker, Dimension FastScan, NCHV-A cantilever) using tapping mode in ambient conditions. The topography data was processed via a custom MATLAB script that performed row alignment, plane levelling, and function fitting to extract structural parameters, RMS roughness, and error values.

The high-resolution (25-35 nm periodicity) square lattices were measured using an AFM (Veeco Dimension V with Nanosensors PPP-NCHR probes) in non-contact mode. To extract a quantitative measure 
of the high-resolution lattice quality, Fourier analysis was used on the measured topography data. The 2D FFT of the topography data revealed prominent peaks (along $k_{x}$ at $k_{y}=0$, and along $k_{y}$ at $k_{x}=0$ ) that correspond to the fundamental spatial frequency of the lattice. The ratio of the fundamental peak height to the next-highest peak in the Fourier spectrum was taken as a quantitative metric for the lattice quality. We chose a threshold of 5 for this ratio as our criterion for a high-quality lattice. The lattice with $29 \mathrm{~nm}$ periodicity was the shortest period that had a ratio greater than $5(5.05)$. Thus, $29 \mathrm{~nm}$ was taken as our limiting spatial resolution.

Transfer of hBN lens. After etching and topography characterization, the hBN lens was transferred to the DBR substrate using a standard dry polymer transfer method ${ }^{\mathrm{S} 4}$ under inert atmosphere in a glove box.

Optical cavity measurements. After the $\mathrm{hBN}$ lens was transferred to the first DBR substrate, another moveable DBR was brought close to the first (distance of $\sim 33 \mu \mathrm{m}$ ), forming an optical microcavity with two planar mirrors. The cavity modes were excited with a broadband source (Fianium supercontinuum laser, NKT Photonics) at a range of incident angles, where the transmitted light was collected and the Fourier plane was spectrally dispersed onto a liquid-nitrogen-cooled charge-coupled-device (CCD) camera (Figure S5a). The control cavity spectra for two planar mirrors without the lens was taken by passing the excitation beam through the flat part of the hBN flake (Figure S5b). In this case, unstable, low- $Q$ longitudinal modes are observed. The cavity spectra for the hBN lens was taken when the excitation beam was passed through the lens, resulting in the cavity transmission spectra shown in Figure $3 \mathrm{f}$ and Figure S5c. To measure the transverse intensity profiles of the different cavity modes, the sample was excited with a narrowband laser (Solstis, M Squared), which is tuned to the energy of the cavity mode of interest. The transverse mode intensity distribution is then recorded on a CCD camera (Chameleon3, FLIR Systems), by imaging the surface of the sample with a microscope with $15 \times$ magnification.

Guided-mode coupling measurements. The hBN flake, optical setup, and optical data used to characterize the in-plane guided-mode coupling to and from the hBN flake are depicted in Figure S6. Two aligned grating couplers, each with a measured period of $282 \mathrm{~nm}$ (Figure S6a), were patterned in an hBN flake of $255 \mathrm{~nm}$ thickness, where the two gratings were separated by $\sim 20 \mu \mathrm{m}$. To observe in-plane guided-mode coupling, we performed measurements where one grating coupler was illuminated to couple light into a guided mode of the flake and the second coupler was used to couple light out of the guided mode. The output was then collected and imaged onto a spectrometer for dispersed $k$-space imaging. ${ }^{55}$ 
The measurements were performed using an inverted optical microscope (Nikon, Eclipse Ti-U) with a $50 \times$ air objective [Nikon, TU Plan Fluor, numerical aperture (NA) of 0.8], imaging both grating couplers simultaneously. As a light source, we used a broadband halogen lamp. A small, circular aperture located in the real-space image plane before the objective ensured that only the first grating coupler was illuminated and that no light was incident on the second grating coupler. After the aperture, the light was reflected onto the sample using a beamsplitter, where it passed through the objective lens and was focused on the sample. Therefore, in this configuration, the first grating coupler was illuminated with broadband light under all possible incident angles (limited by the NA of the objective).

To observe guided-mode coupling, the outcoupled light at the second grating coupler was collected by the same objective, transmitted through the same beamsplitter and passed through another small circular aperture located in the real-space image plane. This second aperture was used to ensure that light was only collected from the second grating coupler on the hBN flake. The back focal plane of the microscope objective was then imaged onto the entrance slit of an imaging spectrograph (Andor Shamrock 303i) and captured by a digital camera (Andor Zyla PLUS sCMOS).

Dispersed $k$-space measurements ${ }^{\mathrm{S} 5}$ were performed by inserting a grating $\left(150\right.$ lines $\mathrm{mm}^{-1}$ blazed at $500 \mathrm{~nm}$ ) into the imaging path of the spectrometer, such that the outcoupled light was spectrally dispersed along one axis of the camera pixel array. A slit was closed to a width of $100 \mu \mathrm{m}$ along the $k_{x}$ axis at $k_{y} \approx 0$. Here, $k_{x}$ is the wavevector direction that corresponds to the modulated direction of the two grating couplers. Thus, the setup allowed for an angle- and wavelength-resolved measurement of the light coupled out at the second grating coupler with a single image. To eliminate the effects of background and stray light incident on the camera, a reference measurement on the flat portion of the same hBN flake was performed and subtracted. Furthermore, a linear polarizer was placed in the collection path to selectively measure s- or p-polarized light. We note that s-polarized light corresponds to a transverse-electric (TE) waveguide mode, and p-polarized light corresponds to a transverse-magnetic (TM) mode. A schematic of the optical setup is shown in Figure S6b.

Figure S6c shows a dispersed $k$-space measurement, where we observe two branches corresponding to outcoupled light that originates from two guided modes of the hBN flake. The lower branch corresponds to the $\mathrm{TE}_{0}$ mode, and the upper branch corresponds to the $\mathrm{TE}_{1}$ mode. We note that the broken inversion symmetry around $k_{x}=0$ occurs because the light is coming from a single direction, starting at the first (incoupling) grating coupler and moving towards the second (outcoupling) grating coupler. We confirmed that if the light 
propagation direction was reversed, the data shown in Figure S6c flipped around $k_{x}=0$. A comparison between measurements for s- and p-polarized light shows that the outcoupled light is s-polarized (TE). This is consistent with the theoretical expectation that, for this wavelength range and flake structure, only the $\mathrm{TE}_{0}$ and $\mathrm{TE}_{1}$ modes of the hBN flake are coupled (see Section S3).

\section{Section S2. Freeform Pattern Design}

Freeform pattern design. All patterns were designed using simple mathematical expressions. The height profile of the surface is represented in Cartesian coordinates for one dimension (1D) as $f(x)$ and in two dimensions (2D) as $f(x, y)$. The spiral phase plates are represented in polar coordinates as $f(r, \theta)$. The origin is located in the middle of the pattern, and the height profiles are defined relative to the unpatterned polymer surface, which is in the $x y$ plane at $z=0 . z$ is orthogonal to the polymer surface with $+z$ pointing away from the substrate.

The Mandelbrot set used in Figure 1 was calculated by iterating the nonlinear equation:

$$
z_{n+1}=z_{n}^{2}+c
$$

where $z_{n}, z_{n+1}$, and $c$ are complex numbers. This equation can be used to generate a 2D image in which each pixel corresponds to a unique value of the complex number $c$, which is then plugged into the iterative equation with a starting value of $z_{0}=0$. The equation is first used to determine $z_{1}$, which is used to determine $z_{2}$, etc. The image of the entire Mandelbot set in Figure S1a was calculated using 500 iterations, where the $x$-axis, $\operatorname{Im}\{c\}$, ranges from 1.3 (left) to -1.3 (right), and the $y$-axis, $\operatorname{Re}\{c\}$, ranges from 1 (bottom) to -2 (top). The image was mapped onto a $1000 \times 1000$ pixel grid. The color scale outside the middle black region denotes the natural logarithm of the number of iterations required for the magnitude of $z_{n+1}$ to be greater than or equal to 2:

$$
f(x, y)=\ln \left(n_{c_{x, y},\left|z_{n+1}\right| \geq 2}\right)
$$

where deep blue (white) is a larger (smaller) number. The black region inside the boundary represents solutions that have a complex magnitude less than 2 after 500 iterations. When the number of iterations is taken to infinity, the values inside the boundary correspond to the Mandelbrot set.

The bitmap in Figure 1b represents a portion of the Mandelbrot set (see red box in Figure S1a). It was calculated using the same procedure as above, except the center of the image is now located at $-0.5135-0.5765 i$, and the $x$-axis, $\operatorname{Im}\{c\}$, ranges from -0.501 (left) to -0.652 (right), and the $y$-axis, $\operatorname{Re}\{c\}$, 
ranges from -0.4264 (bottom) to -0.6006 (top). This portion of the Mandelbrot set is mapped onto a $2000 \times 2000$ pixel grid and corresponds to 100 iterations. Finally, the bitmap is used to control the thermal scanning probe, where the depth of the pattern was chosen to range over approximately $80 \mathrm{~nm}$, from 25 to $105 \mathrm{~nm}$ deep. The lateral size of the pattern was chosen to be $30 \times 30 \mu \mathrm{m}^{2}$ with $15 \times 15 \mathrm{~nm}^{2}$ pixels.

The freeform resolution target in Figure 2a was calculated with the expression:

$$
f(x, y)=-(A-m|x|) \sin \left[(k x)^{3}\right]^{2}-(A-m|y|) \sin \left[(k y)^{3}\right]^{2}-\Delta
$$

where $A$ is the amplitude at the origin, $m$ is the slope that describes the linearly decreasing amplitude away from the origin, $k=2 \pi / \Lambda$ is the spatial frequency at the origin with $\Lambda=12.5 \mu \mathrm{m}$, and $\Delta$ is the vertical offset. The lateral size of the pattern was chosen to be $15.03 \times 8.49 \mu \mathrm{m}^{2}$, mapped onto a $10 \times 10 \mathrm{~nm}^{2}$ pixel grid.

The spiral phase plates in Figure 3a,b were calculated using:

$$
f(r, \theta)=-m \theta-\Delta
$$

where $m$ is the slope describing the linearly decreasing height of the spiral phase plate as a function of the polar angle $\theta$, and $\Delta$ is the vertical offset. The lateral size of an individual phase plate was chosen to be $5 \times 5 \mu \mathrm{m}^{2}$, mapped onto a $10 \times 10 \mathrm{~nm}^{2}$ pixel grid.

The spherical lens in Figure 3c,d was calculated with:

$$
f(x, y)=\sqrt{R^{2}-x^{2}-y^{2}}-\Delta
$$

where $R$ is the radius of curvature of the lens, chosen to be $100 \mu \mathrm{m}$, and $\Delta$ is the vertical offset. The lateral size of the pattern was chosen to be $20.02 \times 20.02 \mu \mathrm{m}^{2}$, mapped onto a $20 \times 20 \mathrm{~nm}^{2}$ pixel grid.

The high-resolution pattern in Figure 2e, the electronic Fourier surfaces in Figure 4, and the large-area pattern in Figure S7 were calculated using:

$$
f(x, y)=\sum_{i} A_{i} \cos \left(k_{i}\left[x \cos \theta_{i}+y \sin \theta_{i}\right]\right)-\Delta
$$

where $A_{i}, k_{i}$, and $\theta_{i}$ correspond to the amplitude, spatial frequency, and in-plane rotation angle, respectively, for component i. $\Delta$ is the vertical offset. For the high-resolution pattern in Figure 2e, the lateral size was $580 \times 580 \mathrm{~nm}^{2}$, mapped onto a $2.9 \times 2.9 \mathrm{~nm}^{2}$ pixel grid. For the electronic Fourier surfaces in Figure 4, the lateral size was $1 \times 1 \mu \mathrm{m}^{2}$, mapped onto a $5 \times 5 \mathrm{~nm}^{2}$ pixel grid. For the large-area electronic Fourier surface in Figure $\mathrm{S} 7$, the lateral size was $10 \times 10 \mu \mathrm{m}^{2}$, mapped onto a $5 \times 5 \mathrm{~nm}^{2}$ pixel grid.

The photonic grating couplers in Figure S6 were calculated using:

$$
f(x)=A \cos (k x)-\Delta
$$


where $A$ and $k$ correspond to the amplitude and spatial frequency, respectively. $\Delta$ is the vertical offset. The lateral size was $14 \times 14 \mu \mathrm{m}^{2}$, mapped onto a $10 \times 10 \mathrm{~nm}^{2}$ pixel grid. For the parameters used in all formulas in this section, see Table S1.

Bitmap generation. The mathematical expressions were converted into bitmaps, where the overall dimensions for the structure were chosen and the pattern was mapped onto a pixel grid (see above). The normalized depth of the pattern in the $z$-direction was discretized into 256 levels, corresponding to 8-bit precision. The physical depth of the patterns was assigned during patterning with the thermal scanning probe, where the total pattern depth was taken as an input, and the thermal scanning-probe software mapped the total depth onto the 8-bit depth levels.

Design rules for in-plane resolution versus pattern depth. The conical shape of the thermal scanning probe, combined with mechanical deformations in the polymer resist, set the lower limit on in-plane periodicity for a given depth. This limit can be estimated as follows. A fresh probe has a tip diameter at the apex as low as $6 \mathrm{~nm}$ and half-angle of $15-30^{\circ}$. We note that these quantities vary from probe to probe due to fabrication tolerances. Thus, the probe width is a function of the distance from the tip, set by the pattern depth. For a periodic structure, the relationship between the minimum periodicity, $\Lambda_{\min }$, and the pattern depth, $d$, can be written as:

$$
\Lambda_{\text {min }}=2 w(d)=2\left(w_{0}+2 d \tan \theta_{\text {half }}+w_{m}\right)
$$

where $w(d)$ is the width of the indent, $w_{0}$ is the probe width at the apex, $\theta_{\text {half }}$ is the opening half-angle of the probe tip, and $w_{m}$ represents additional feature broadening beyond the probe shape due to mechanical deformations. A detailed discussion of this topic is available. ${ }^{\mathrm{S} 6}$ The prefactor of 2 arises from the assumption that a periodic structure will have a period twice as wide as the indent caused by the probe. We note that in practice contamination will increase the size of the probe, which can further increase $\Lambda_{\min }$. Furthermore, roughness accumulated during etching can additionally increase $\Lambda_{\min }$ for the final pattern in hBN.

\section{Section S3. Calculation of Optical Modes}

Modeling of cavity modes. The frequencies of the different transverse and longitudinal modes of the microlens cavity are given by the resonant standing-wave condition ${ }^{\mathrm{S} 7}$

$$
\omega_{q m n}=\frac{c}{2 * L_{\mathrm{eff}}}\left\{q+(n+m+1) \frac{\cos ^{-1}\left[ \pm \sqrt{g_{1} g_{2}}\right]}{\pi}\right\}
$$

where $\{q, n, m\}$ is a set of integers labelling the longitudinal and transverse mode orders, respectively, $L_{\text {eff }}$ is the effective cavity length, $g_{1}=1$ for the flat DBR mirror, and 


$$
g_{2}=1-\left(n_{\mathrm{hBN}}-1\right) * L_{\mathrm{eff}} / r_{\mathrm{hBN}}
$$

for the DBR mirror with the $\mathrm{hBN}$ lens. The refractive index of $\mathrm{hBN}, n_{\mathrm{hBN}}$, was set to 2.12 (ref. S8), and the radius of curvature of the microlens was set to $r_{\mathrm{hBN}}=95 \mu \mathrm{m}$, according to our AFM measurement. As shown in Figure S5, this simple analytical solution to the resonator mode equation reproduces the measured spectrum. We note that the lens has a diameter of $\sim 10 \mu \mathrm{m}$ and a numerical aperture (NA) of 0.05 .

Theoretical calculations of the guided modes. To compare the observed guided modes to theoretical predictions, we performed electromagnetic simulations (Lumerical). We considered a system with an hBN layer that is surrounded by an infinite half-space of air above and an infinite half-space of $\mathrm{SiO}_{2}$ below. For the $\mathrm{hBN}$ layer we used the measured thickness of $255 \mathrm{~nm}$ and the previously measured in-plane and out-of-plane indices of refraction. ${ }^{\mathrm{S} 8}$ Calculating the eigenmodes for the experimental wavelength range and their corresponding effective indices, $n_{\text {eff }}\left(\lambda_{0}\right)$, yields the effective wavevector $k_{\text {eff }}=n_{\text {eff }}\left(2 \pi / \lambda_{0}\right)$ of the incoupled light propagating inside the waveguide as a function of the free-space wavelength $\lambda_{0}$. The outcoupler grating then couples light from the guided mode to light in free space with an in-plane momentum according

to $k_{x, \text { out }}=k_{\text {eff }}-k_{\mathrm{g}}$. Here, $k_{\mathrm{g}}=\frac{2 \pi}{\Lambda_{\mathrm{g}}}$ is the wavevector associated with the period of the grating coupler with $\Lambda_{\mathrm{g}}$ equal to $282 \mathrm{~nm}$. Therefore, each guided mode in the measurements of Figure S6c will appear as a branch that follows the dispersion relation $E(k)=\hbar c k_{\mathrm{eff}}$ but at an in-plane momentum shifted by $-k_{\mathrm{g}}$ into the light cone. Here, $\hbar$ is the reduced Planck constant and $c$ is the speed of light in vacuum. Figure S6c shows the theoretical expectation of the $\mathrm{TE}_{0}$ and $\mathrm{TE}_{1}$ modes obtained from our numerical calculations. The shaded regions correspond to a $\pm 3 \%$ uncertainty on the reported indices of refraction for $\mathrm{hBN} .^{\mathrm{S} 8}$

\section{Section S4. Simulation of Electrostatic Potentials}

Electrostatic simulations. Electrostatic simulations of devices incorporating patterned hBN structures are performed by solving Poisson's equation with the finite-element solver COMSOL. The devices consisted of an upper and lower hBN flake that together encapsulated a 2D active layer in between (Figure S8a). The lower $\mathrm{hBN}$ flake has a thickness of $10 \mathrm{~nm}$ and is flat. The upper hBN flake was structured such that the top surface had a depth modulation of $20 \mathrm{~nm}$ and a $5 \mathrm{~nm}$ spacing between the bottom of the pattern and the bottom surface of the flake. Furthermore, we assumed an isotropic dielectric constant for hBN with a value of 4.5 and considered a potential difference of $1 \mathrm{~V}$ between the top and bottom gates, represented by the top and bottom surfaces of the two hBN flakes, respectively. 
Section S5. Table of Experimental Design Parameters for Freeform Nanostructures

\begin{tabular}{|c|c|c|c|c|c|c|c|c|c|c|c|c|c|c|}
\hline \multirow[b]{2}{*}{ Figure } & \multicolumn{5}{|c|}{ Parameters } & \multicolumn{9}{|c|}{ Height profile } \\
\hline & $\begin{array}{c}A \\
(\mathrm{~nm})\end{array}$ & $\begin{array}{c}m \\
\left(\mathrm{~nm} \mu \mathrm{m}^{-1}\right)\end{array}$ & $\begin{array}{c}\Lambda \\
(\mu \mathrm{m})\end{array}$ & $s$ & $\begin{array}{c}\Delta \\
(\mathrm{nm})\end{array}$ & \multicolumn{9}{|c|}{$f(x, y)$} \\
\hline Fig. 2a & 28.5 & 3.7 & 12.5 & 2 & 0 & \multirow{3}{*}{\multicolumn{9}{|c|}{$-(A-m|x|) \sin \left[(k x)^{3}\right]^{s}-(A-m|y|) \sin \left[(k y)^{3}\right]^{s}-\Delta$}} \\
\hline Fig. $2 b$ & 42.8 & 5.5 & 12.5 & 2 & 0 & & & & & & & & & \\
\hline Fig. 2d & 2.5 & 0 & 12.5 & 1 & 2.5 & & & & & & & & & \\
\hline Figure & $i$ & $\begin{array}{c}A_{i} \\
(\mathrm{~nm})\end{array}$ & $\begin{array}{c}\Lambda_{i} \\
(\mathrm{~nm})\end{array}$ & $\begin{array}{c}\theta_{1} \\
(\operatorname{deg})\end{array}$ & \begin{tabular}{|c}
$\theta_{2}$ \\
$(\operatorname{deg})$
\end{tabular} & $\begin{array}{c}\theta_{3} \\
(\operatorname{deg})\end{array}$ & $\begin{array}{c}\theta_{4} \\
(\mathrm{deg})\end{array}$ & $\begin{array}{c}\theta_{5} \\
(\mathrm{deg}) \\
\end{array}$ & $\begin{array}{c}\theta_{6} \\
(\mathrm{deg})\end{array}$ & $\begin{array}{c}\theta_{7} \\
(\operatorname{deg})\end{array}$ & $\begin{array}{c}\theta_{8} \\
(\mathrm{deg})\end{array}$ & $\begin{array}{c}\theta_{9} \\
(\operatorname{deg})\end{array}$ & $\begin{array}{c}\Delta \\
(\mathrm{nm})\end{array}$ & $f(x, y)$ \\
\hline Fig. $2 \mathrm{e}$ & 1,2 & 1.9 & 29 & 0 & 90 & - & - & - & - & - & - & - & 3.8 & \multirow{6}{*}{$\sum_{i} A_{i} \cos \left[k_{i}\left(x \cos \theta_{i}+y \sin \theta_{i}\right)\right]-\Delta$} \\
\hline Fig. $4 a$ & $\begin{array}{c}1,2 \\
3\end{array}$ & 2.2 & 50 & 0 & 60 & 120 & - & - & - & - & - & - & 6.7 & \\
\hline Fig. $4 d$ & $1-6$ & 1.1 & 50 & 0 & 60 & 120 & 10 & 70 & 130 & - & - & - & 6.7 & \\
\hline Fig. $4 \mathrm{~g}$ & $1-6$ & 1.1 & 47,55 & 0 & 60 & 120 & 0 & 60 & 120 & - & - & - & 6.7 & \\
\hline Fig. $4 \mathrm{j}$ & $1-9$ & 0.8 & 50 & 0 & 20 & 40 & 60 & 80 & 100 & 120 & 140 & 160 & 6.9 & \\
\hline Fig. S7 & $\begin{array}{c}1,2 \\
3\end{array}$ & 2.2 & 50 & 0 & 60 & 120 & - & - & - & - & - & - & 6.7 & \\
\hline Figure & \multicolumn{7}{|c|}{$\begin{array}{c}m \\
\left(\mathrm{~nm} \mathrm{rad}^{-1}\right)\end{array}$} & \multicolumn{6}{|c|}{$\begin{array}{c}\Delta \\
(\mathrm{nm})\end{array}$} & $f(r, \theta)$ \\
\hline Fig. 3b & \multicolumn{7}{|c|}{18.3} & \multicolumn{6}{|c|}{10.0} & $-m \theta-\Delta$ \\
\hline Figure & \multicolumn{7}{|c|}{$\begin{array}{c}R \\
(\mu \mathrm{m})\end{array}$} & \multicolumn{6}{|c|}{$\underset{(\mu \mathrm{m})}{\Delta}$} & $f(x, y)$ \\
\hline Fig. 3d & \multicolumn{7}{|c|}{100} & \multicolumn{6}{|c|}{100.0} & $\sqrt{R^{2}-x^{2}-y^{2}}-\Delta$ \\
\hline Figure & \multicolumn{3}{|c|}{$\begin{array}{c}A \\
(\mathrm{~nm})\end{array}$} & \multicolumn{4}{|c|}{$\begin{array}{c}\Lambda \\
(\mathrm{nm})\end{array}$} & \multicolumn{6}{|c|}{$\begin{array}{c}\Delta \\
(\mathrm{nm})\end{array}$} & $f(x)$ \\
\hline Fig. S6 & \multicolumn{3}{|c|}{20.0} & \multicolumn{4}{|c|}{280} & \multicolumn{6}{|c|}{20.0} & $A \cos (k x)-\Delta$ \\
\hline
\end{tabular}

Table S1. Design parameters for freeform nanostructures. The design parameters for freeform nanostructures are based on analytical functions. The functions are defined for the pattern in the polymer surface, written by the thermal scanning probe, where $x$ and $y$ lie in the sample plane and $z$ is perpendicular, pointing away from the substrate. A right-handed coordinate system is used. The origin is placed in the middle of the pattern in the $x$ and $y$ directions, and at the unpatterned polymer surface in the $z$ direction. The height of the patterned surface is defined relative to the unpatterned polymer surface at $z=0$. The parameters $A$ and $\Delta$ have been rounded to the nearest $0.1 \mathrm{~nm}$, and $m$ has been rounded to the nearest $0.1 \mathrm{~nm} \mu \mathrm{m}^{-1}$ and $0.1 \mathrm{~nm} \mathrm{rad}^{-1}$. 


\section{Section S6. Supplementary Figures}
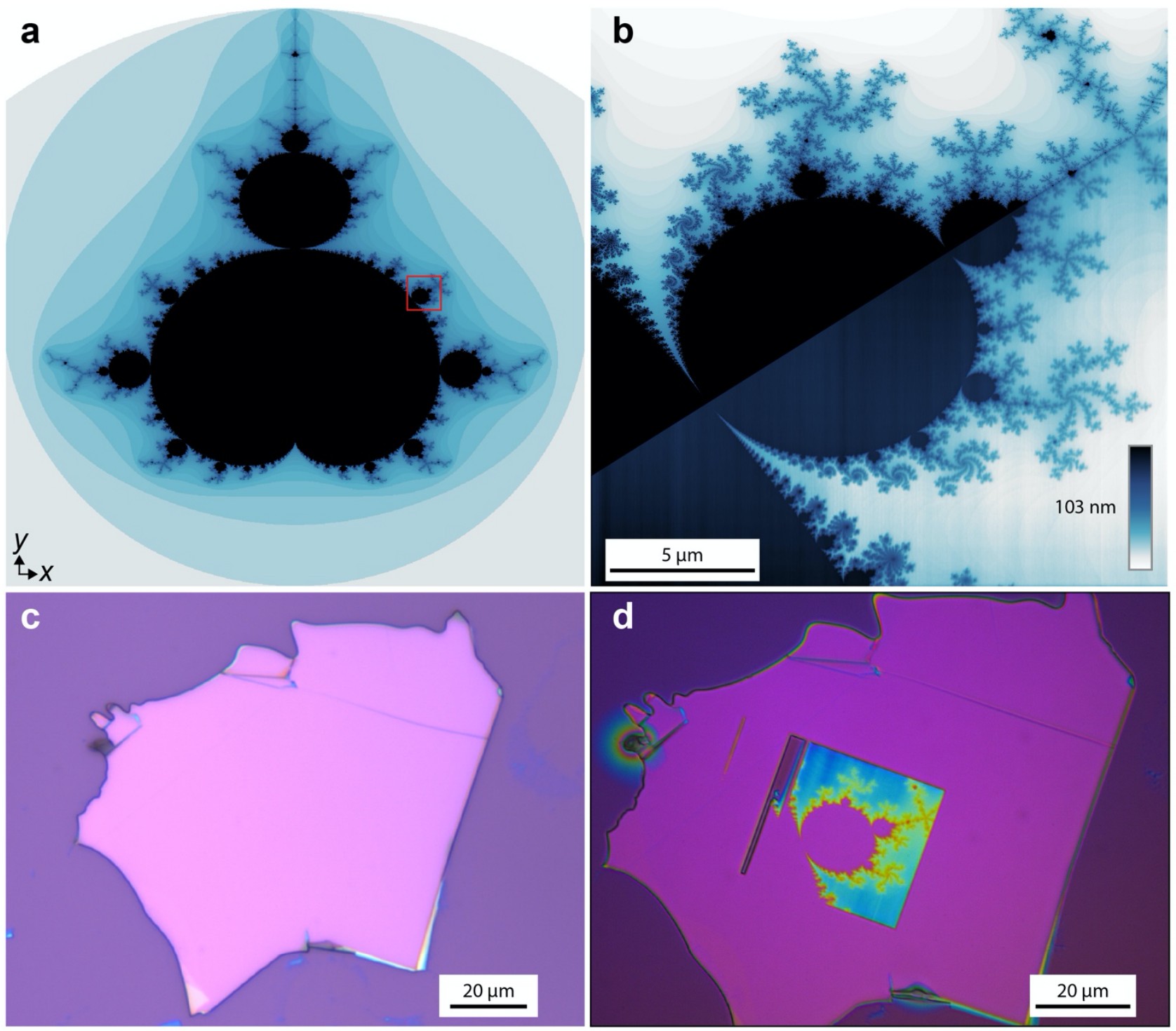

Figure S1. Design and fabrication of a Mandelbrot set pattern in an hBN flake. (a) Full Mandelbrot bitmap. The red box indicates the portion of the pattern used in Figure 1. (b) Portion of the Mandelbrot set used in Figure 1 (red box in panel a). Top left: bitmap. Bottom right: topography data of the polymer measured by the thermal scanning probe during patterning. (c) Optical microscope image of the $\mathrm{hBN}$ flake $(\sim 126 \mathrm{~nm}$ thick) before the polymer resist was spun on top. (d) Optical microscope image of the $\mathrm{hBN}$ flake from panel $\mathrm{c}$ patterned with a portion of the Mandelbrot set (red box in panel a). The image was taken after etching and cleaning, where all residual polymer is assumed to be removed. 

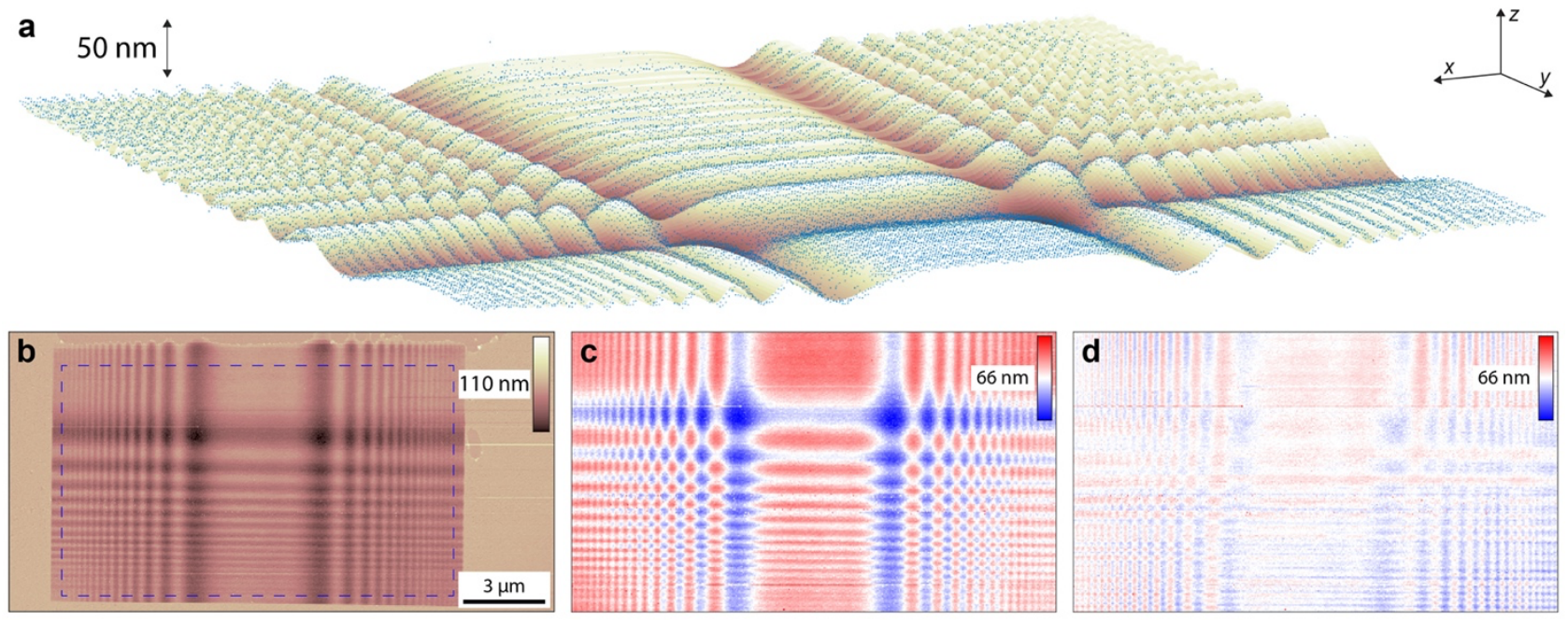

Figure S2. Topography characterization of hBN freeform resolution target. (a) Measured topography (AFM, blue points) and fitted function (yellow/brown surface) of the freeform resolution target in hBN (Figure 2a). (b) Measured topography (AFM) of the freeform resolution target in hBN. The dashed blue box indicates the region used for fitting the 2D freeform function. (c) Measured topography of the structure in panel b, plotted only for the fit region (blue box in panel b), scaled from the minimum depth value to the maximum depth value and centered at zero. (d) Residual error between the data and fitted function, plotted for the fit region as in panel c. For comparison, the data is scaled over the same range as in panel c, centered at zero. For all structural design parameters, see Table S1. 

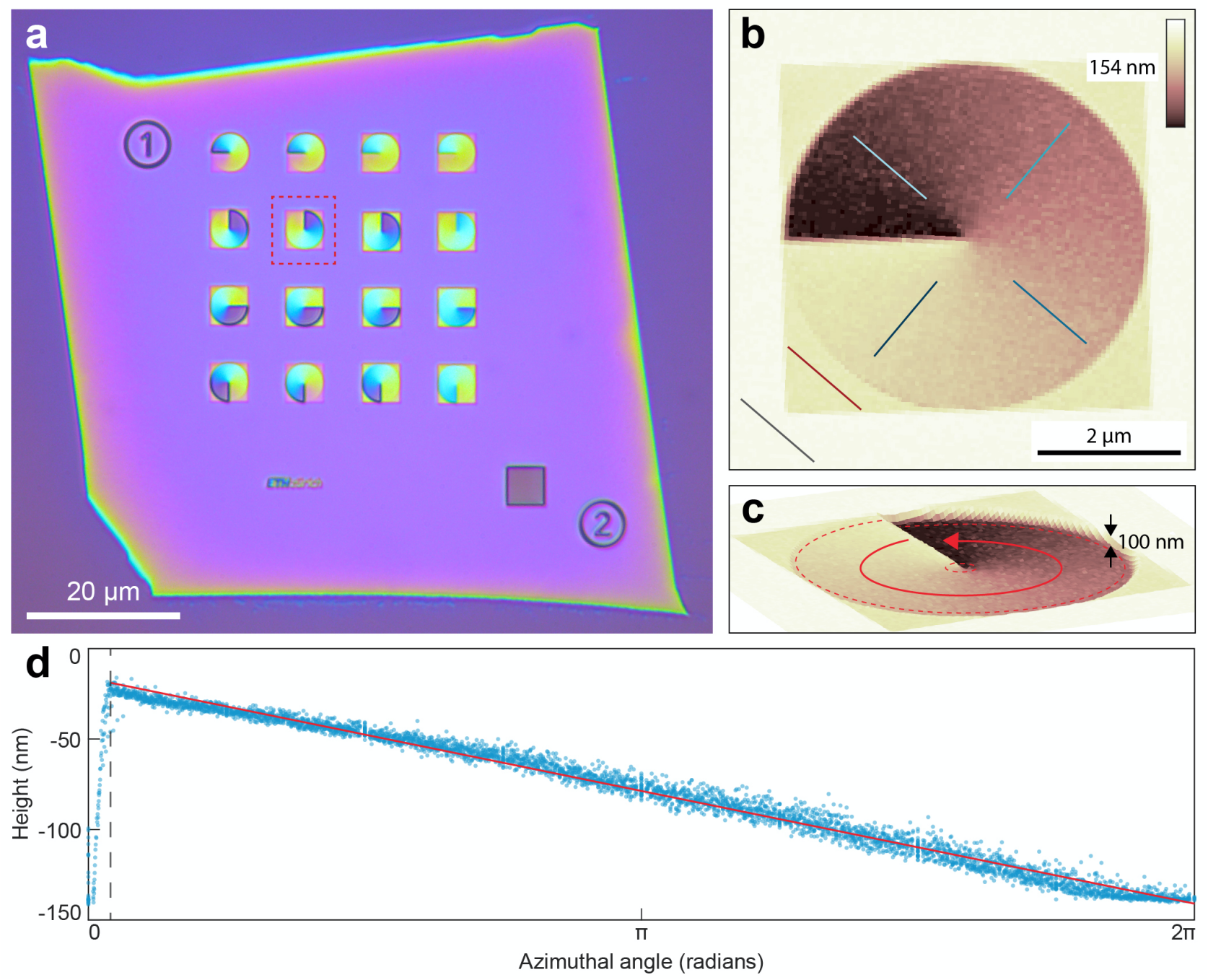

Figure S3. Topography characterization of hBN spiral phase plate. (a) Optical microscope image of the hBN flake from Figure 3a,b ( $\sim 130 \mathrm{~nm}$ thick) containing an array of spiral phase plates. These structures will impart a spiral $2 \pi$ phase modulation on deep-ultraviolet photons at $\sim 200 \mathrm{~nm}$. (b) Measured topography (AFM) of the phase plate indicated with the dashed red box in panel a. The overlaid lines (grey, red, and series of blue) indicate line cuts used to determine the RMS roughness of the unpatterned flake $(0.4 \mathrm{~nm})$, of a flat region on the patterned flake $(0.8 \mathrm{~nm})$, and of the freeform pattern $(1.7 \mathrm{~nm})$, respectively. (c) Same data as in panel b, shown as a three-dimensional topography plot. (d) Measured topography (AFM, blue points, collected from the annular region between the dashed red lines in panel c) and fitted function (red line) of the spiral phase plate, expressed as a linear function in polar coordinates. The linear fit returns an RMS error of $4.0 \mathrm{~nm}$ for a pattern depth of $115 \mathrm{~nm}$ (3.5\%). For all structural design parameters, see Table S1. 

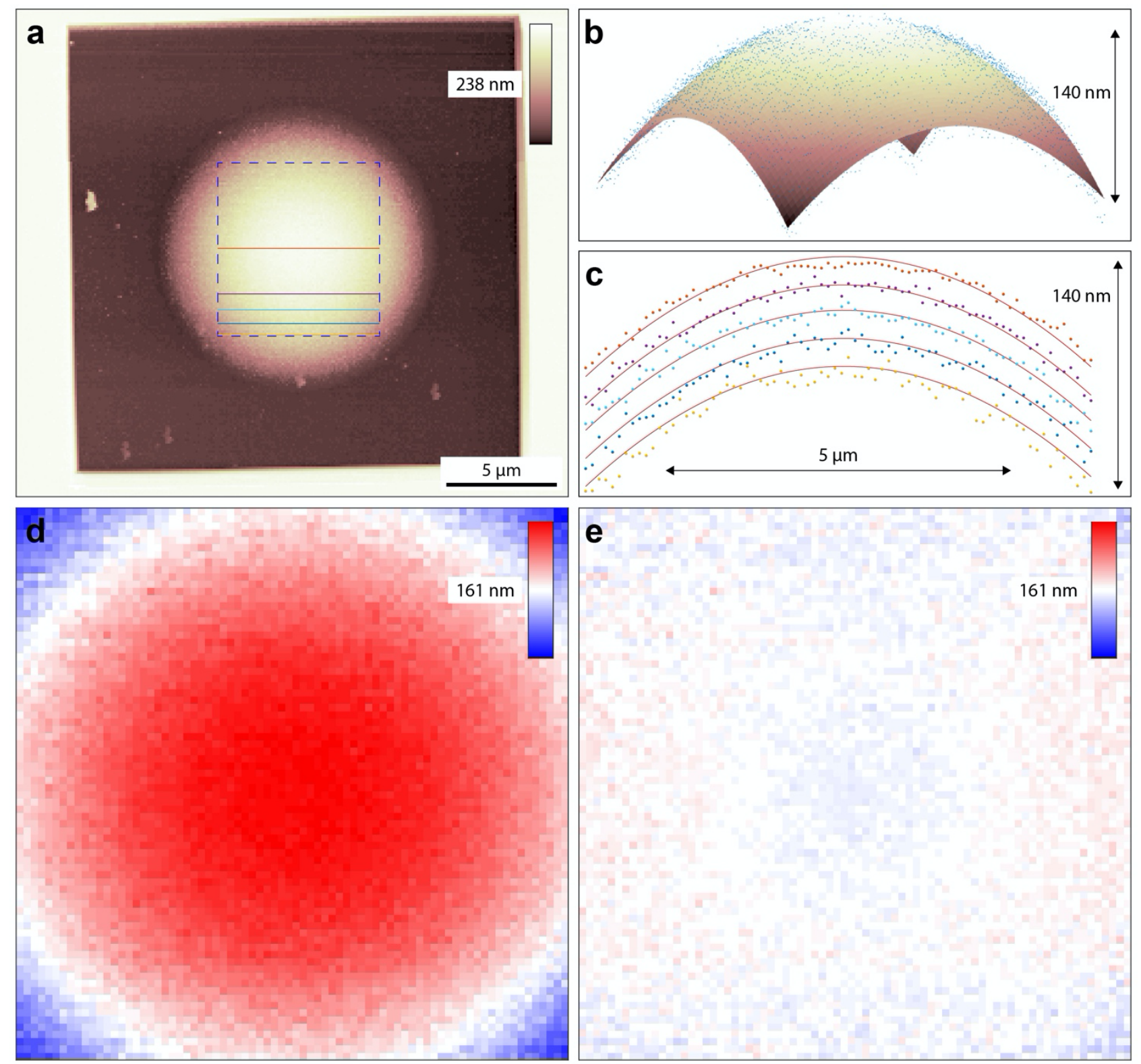

Figure S4. Topography characterization of hBN microlens. (a) Measured topography (AFM) of the spherical hBN microlens in Figure $3 \mathrm{c}, \mathrm{d}$. The dashed blue box indicates the region used for fitting the 2D spherical function. The colored horizontal lines indicate line cuts shown in panel c. (b) Measured topography (AFM, blue points) and fitted function (yellow/brown surface) of the hBN microlens, resulting in an RMS error of $4.5 \mathrm{~nm}(2.8 \%)$. (c) Line cuts (red) through the 2D fitted function, plotted with the corresponding line scans of the measured topography in panel a (points colored to match the horizontal lines in panel a). (d) Measured topography of the structure in panel a, plotted only for the fit region (dashed blue box in panel a), scaled from the minimum depth value to the maximum depth value and centered at zero. (e) Residual error between the data and fitted function, plotted for the fit region as in panel d. For comparison, the data is scaled over the same range as in panel d, centered at zero. For all structural design parameters, see Table S1. 

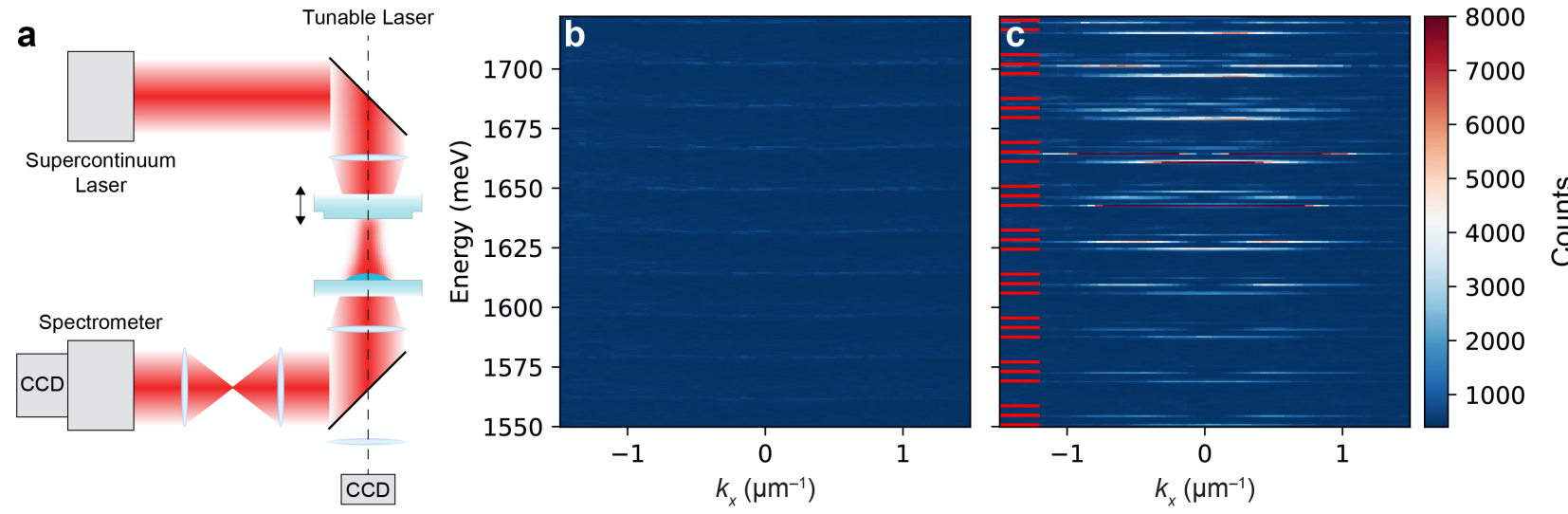

Figure S5. Measurement and modeling of microcavity modes. (a) Diagram of measurement setup, showing the hBN microlens from Figure 3c,d placed between two cavity mirrors. The black arrows indicate that one mirror is movable along the optical axis, where the position is varied to form a tunable microcavity. For the angle-resolved transmission spectra shown in panels $\mathrm{b}$ and $\mathrm{c}$, and Figure $3 \mathrm{f}$, the cavity is fed with a broadband source (supercontinuum laser) at a range of incident angles. A $4 f$ imaging setup (where $f$ is the focal length of the optics) is used to measure angle-resolved cavity transmission, which is spectrally dispersed by a spectrometer and then imaged on a liquid-nitrogen-cooled CCD camera. For the measured transverse-mode profiles shown in Figure 3h, a narrowband tunable laser was used as the source, and the transmitted light was imaged onto a CCD. (b) Angle-resolved transmission spectra (energy versus inplane wavevector, $k_{x}$ ) for a cavity with a flat hBN flake, revealing unstable cavity modes supported by the two planar mirrors. (c) Angle-resolved transmission spectra (as in panel b) for a cavity that includes the hBN microlens. The red lines (left) indicate calculated mode energies. 

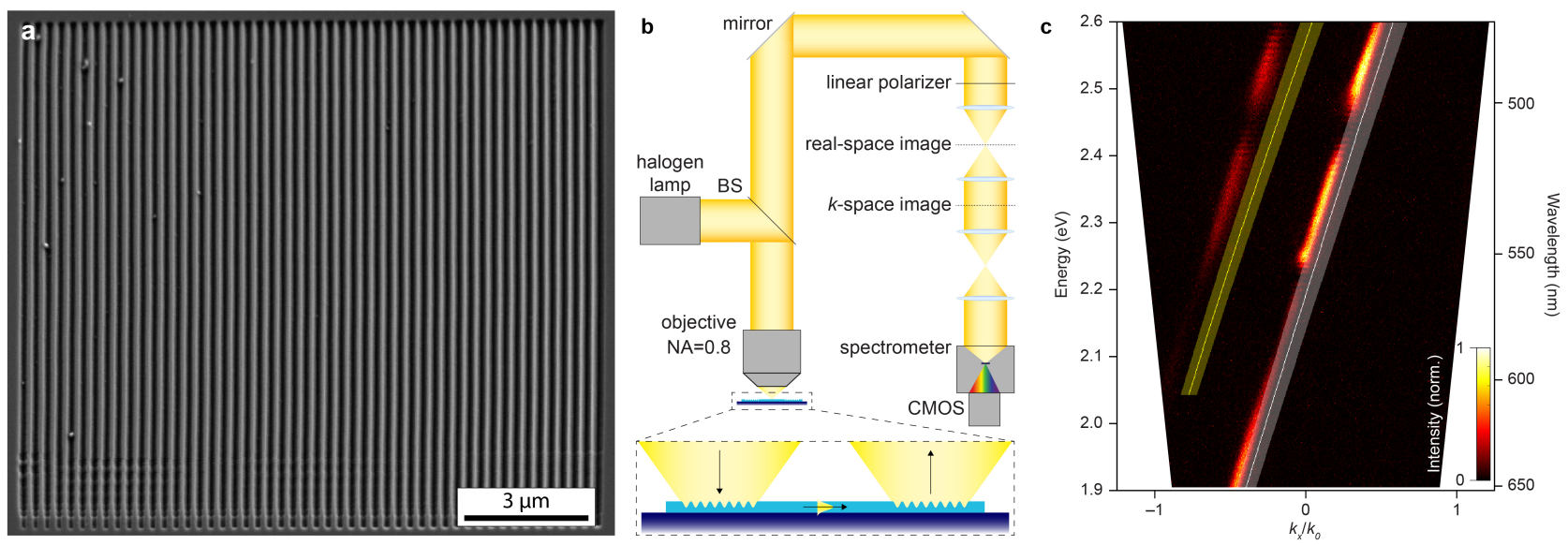

Figure S6. hBN grating couplers. (a) SEM image of a sinusoidal grating coupler patterned in an hBN flake (255 nm thick). (b) Schematic of the optical setup used for the guided-mode coupling measurements. The setup is used to couple broadband light into a guided-mode in the hBN with the first grating. The mode then propagates toward the second grating and is outcoupled. The outcoupled light is analyzed in $k$-space (Section S1). (c) Angle-resolved spectra (energy versus in-plane wavevector, $k_{x}$ with $k_{y} \approx 0$ ) for light outcoupled from the second grating, measured using the setup in panel $\mathrm{b}$. The wavevector is relative to $k_{0}=2 \pi / \lambda_{0}$, where $\lambda_{0}$ is the free-space wavelength of the photon. The red streaks are the experimental data. The white (yellow) line shows the calculated dispersion curve for the $\mathrm{TE}_{0}\left(\mathrm{TE}_{1}\right)$ mode, diffracted into the light cone by the grating. The shaded regions represent a $\pm 3 \%$ uncertainty on the reported indices of refraction for hBN (ref. S8). For all structural design parameters, see Table S1. 


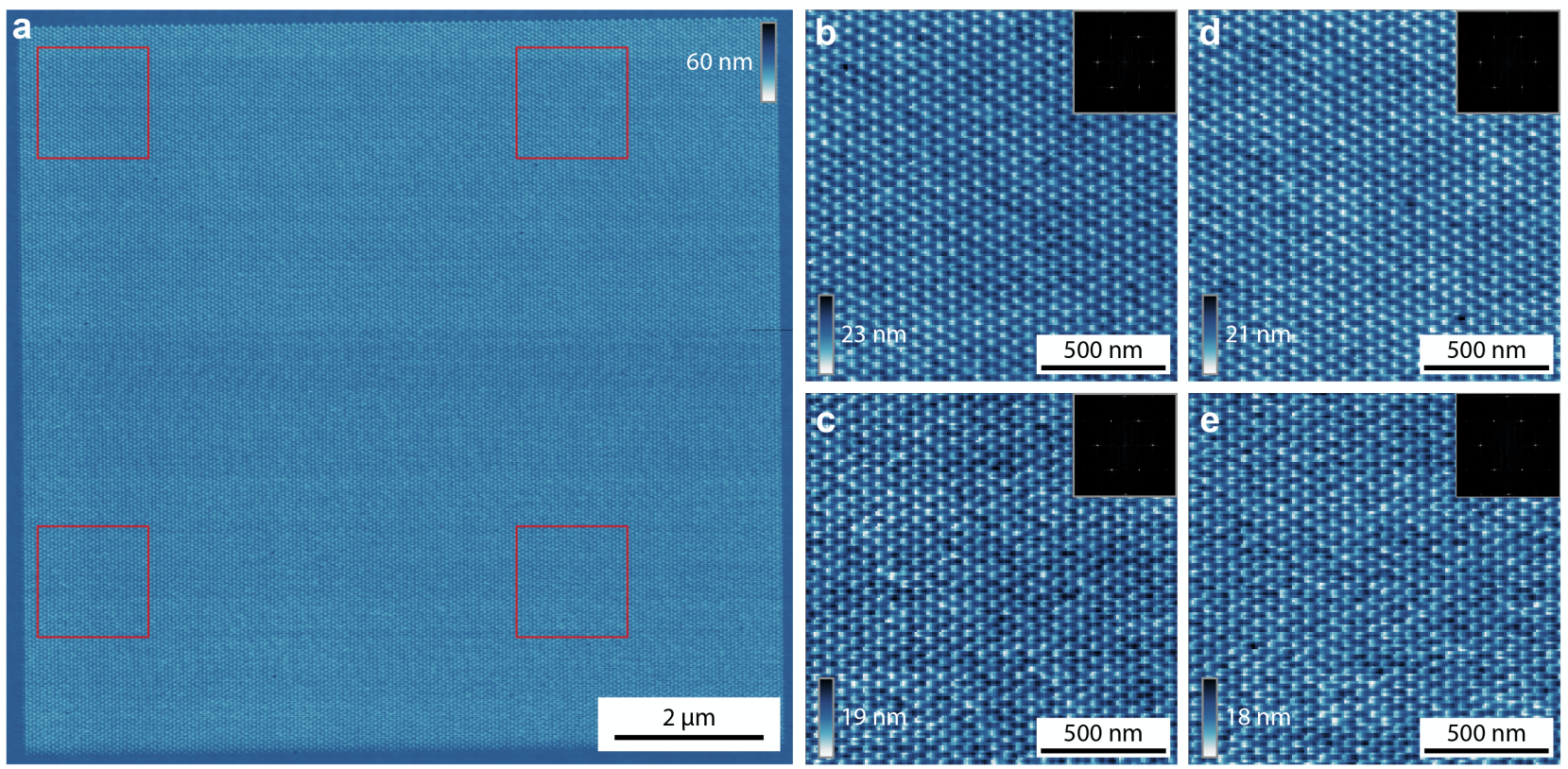

Figure S7. Large-area electronic Fourier surfaces in hBN. (a) Measured topography (AFM) of a $10 \times 10 \mu \mathrm{m}^{2}$ hexagonal electronic Fourier surface in hBN, defined with three sinusoids with a period of $50 \mathrm{~nm}$. (b)-(e) Magnified regions from panel a, revealing a high-quality structure over the entire pattern area. The regions are marked with red boxes in panel a. Insets show FFTs for each pattern. For all structural design parameters, see Table S1. 

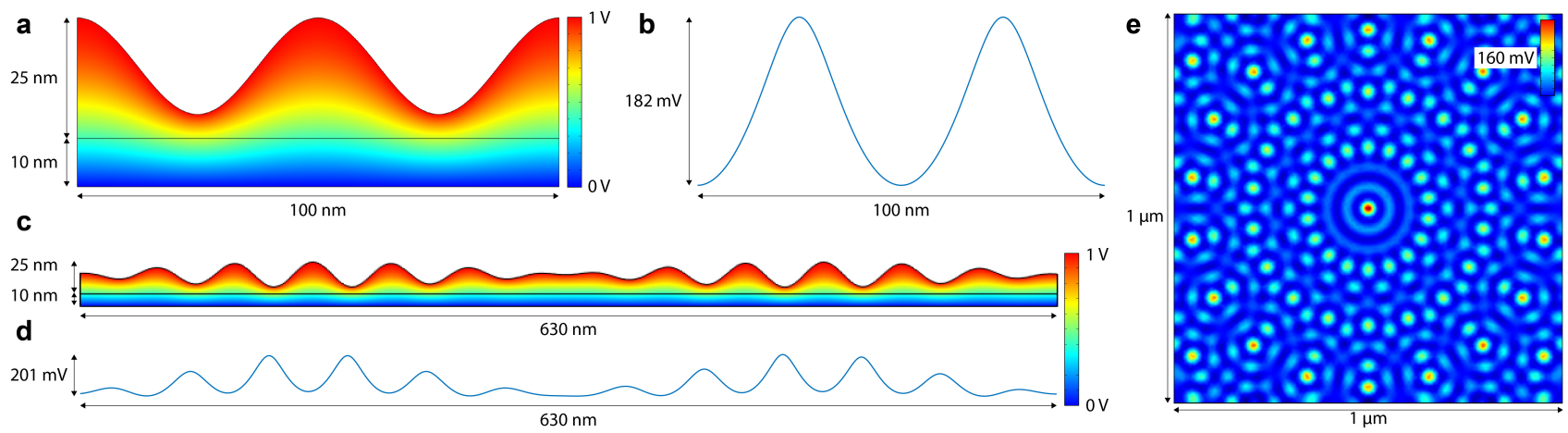

Figure S8. Electrostatic simulations of a 2D layer surrounded by hBN. (a) Simulation of a single-sinusoidal electronic Fourier surface in hBN with a period of $50 \mathrm{~nm}$. The top $\mathrm{hBN}$ has a thickness of $25 \mathrm{~nm}$ with a $20 \mathrm{~nm}$ depth modulation. The bottom hBN has a thickness of $10 \mathrm{~nm}$ and is unstructured. The thin horizontal black line in between the two hBN layers represents the active layer, which could be a monolayer such as $\mathrm{MoS}_{2}$. A voltage of $1 \mathrm{~V}$ is applied to the top surface, representing a top gate, and a voltage of $0 \mathrm{~V}$ is applied to the bottom surface, representing a back gate. The color map represents the electric potential at every point throughout the structure. (b) Simulated electric-potential profile at the active layer (thin black horizontal line in panel a) when $1 \mathrm{~V}$ is applied to the structure in panel a. The surface profile in the top $\mathrm{hBN}$ is revealed in the field profile. (c),(d) As in panels a and b, but for a surface with two sinusoids with periods of 55 and $47 \mathrm{~nm}$. (e) Simulation of the electric-potential profile at the active layer, as in panels b and d, but for a 3D simulation of the quasicrystal structure from Figure 4l, defined with 9 sinusoids, each with $50 \mathrm{~nm}$ period. For all structural design parameters, see Table S1. 


\section{Section S7. Supplementary References}

S1. Novoselov, K. S.; Geim, A. K.; Morozov, S. V.; Jiang, D.; Zhang, Y.; Dubonos, S. V.; Grigorieva, I. V.; Firsov, A. A. Electric Field Effect in Atomically Thin Carbon Films. Science 2004, 306, 666-669.

S2. Huang, Y.; Sutter, E.; Shi, N. N.; Zheng, J.; Yang, T.; Englund, D.; Gao, H.-J.; Sutter, P. Reliable Exfoliation of Large-Area High-Quality Flakes of Graphene and Other Two-Dimensional Materials. ACS Nano 2015, 9, 1061210620.

S3. Grenadier, S.; Li, J.; Lin, J.; Jiang, H. Dry Etching Techniques for Active Devices Based on Hexagonal Boron Nitride Epilayers. J. Vac. Sci. Technol. A 2013, 31, 061517.

S4. Wang, L.; Meric, I.; Huang, P. Y.; Gao, Q.; Gao, Y.; Tran, H.; Taniguchi, T.; Watanabe, K.; Campos, L. M.; Muller, D. A.; Guo, J.; Kim, P.; Hone, J.; Shepard, K. L.; Dean, C. R. One-Dimensional Electrical Contact to a TwoDimensional Material. Science 2013, 342, 614-617.

S5. Kurvits, J. A.; Jiang, M.; Zia, R. Comparative Analysis of Imaging Configurations and Objectives for Fourier Microscopy. J. Opt. Soc. Am. A 2015, 32, 2082-2092.

S6. Ryu Cho, Y. K.; Rawlings, C. D.; Wolf, H.; Spieser, M.; Bisig, S.; Reidt, S.; Sousa, M.; Khanal, S. R.; Jacobs, T. D. B.; Knoll, A. W. Sub-10 Nanometer Feature Size in Silicon Using Thermal Scanning Probe Lithography. ACS Nano 2017, 11, 11890-11897.

S7. Siegman, A. E., Lasers. University Science Books: Mill Valley, 1986.

S8. Rah, Y.; Jin, Y.; Kim, S.; Yu, K. Optical Analysis of the Refractive Index and Birefringence of Hexagonal Boron Nitride from the Visible to Near-Infrared. Opt. Lett. 2019, 44, 3797-3800. 